\title{
Einleitung: Ost-westliche Kontroverstheologie im 13. Jahrhundert
}

Im ökumenischen Fokus der Kirchengeschichtsschreibung werden die Ereignisse des Jahres 1054 längst als ein symbolisches Datum des Schismas zwischen Ost- und Westkirche angesehen. Was noch in vielen kirchengeschichtlichen Studien der zweiten Hälfte des 20. Jahrhunderts als Bruchstelle, ja als ausschlaggebendes Moment oder jedenfalls als Wendepunkt im Auseinanderdriften derjenigen beiden Kirchen gesehen und gelehrt wurde, die wir heute als katholische und orthodoxe Kirche konfessionell voneinander unterscheiden, ${ }^{1}$ weicht mittlerweile in der historischen Beurteilung vielerorts und zu Recht dem vierten Kreuzzug, der Einnahme Konstantinopels und dem Lateinischen Kaiserreich als Brennpunkte des Schismas. Sooft das Datum der gegenseitigen Exkommunikationen von 1054 nun als ,symbolisches“ betont wird, womit nicht selten seine Relevanz im Kontext der (kirchen-)politischen lateinischgriechischen Beziehungen geschmälert wird, sooft wird gleichzeitig übersehen, dass die Akteure von 1054 eben nicht nur Kirchenpolitik betrieben und kirchenrechtlich agierten: Sie waren Theologen, die literarisch-theologisch über den Konfliktstoff reflektierten - ein Konfliktstoff, der sie in ihren Argumentationen und Handlungen antrieb, wie in den Quellen nachvollziehbar ist. Und obwohl - oder vielleicht gerade weil - der kirchenpolitische/-rechtliche Aspekt in der Rezeption von 1054 eindeutig den Vorrang erhalten hat, eröffnet die literarisch-theologische Tätigkeit der Akteure als Initialzündung kontroverstheologischer Auseinandersetzungen den Blick in eine ganz andere Dimension der ost-westlichen Beziehungen, die exemplarisch nachzuverfolgen und $\mathrm{zu}$ analysieren Gegenstand der vorliegenden Studie ist.

Mit dem Brief des Patriarchen von Konstantinopel Michael Kerullarios an den Patriarchen Petros von Antiochia ${ }^{2}$, verfasst im Jahr 1054, beginnt eine Tradition sogenannter Irrtumslisten oder Irrtumskataloge auf Seiten der Byzantiner gegen die Lateiner. Der im selben Jahr entstandene Brief Adversus Graecorum calumnias ${ }^{3}$ des Kardinallegaten Humbert von Silva Candida reiht sich ein in eine bereits bestehende Tradition polemischer Werke unterschiedlicher Gattungen gegen die Byzantiner auf Seiten der Lateiner. Diese beiden Werke sind Beispiel und Modell des Umgangs mit dem jeweils Anderen bzw. mit der anderen Kirche im Spiegel der eigenen Rechtgläubigkeit. Dabei spielt der Rekurs auf den griechischen Patriarchen Photius, jene schillernde Gestalt der ost-westlichen Kontroverse des 9. Jahrhunderts, eine für die Entstehung der polemischen Textgattungen maßgebliche, weil konstitutive Rolle: Die theologische Kritik des Photius in Bezug auf das Filioque und der Appell des Papstes

1 Vgl. die bibliographischen Angaben bei BAYER 22004, 2-7 et passim. Im Bereich research to public findet sich sogar aktuell die inzwischen überholte These von 1054 als entscheidender Bruch zwischen Ost- und Westkirche: vgl. dazu den Beitrag von KIRCHSCHLÄGER 2020, 31-33, hier: 33.

2 Vgl. PG 120, col. 781B - 796 A bzw. WILL 1861, 172-184.

3 Vgl. PL 143, col. 929 - 974 A bzW. WiLl 1861, 93-126.

Ә OpenAccess. (c) 2020 Andrea Riedl, publiziert von De Gruyter. (cc)BY Dieses Werk ist lizenziert unter der Creative Commons Attribution 4.0 International. https://doi.org/10.1515/9783110697667-002 
Nikolaus I. an die westlichen Theologen, entsprechende Gegenentwürfe zu erstellen, gilt allgemein als die „Geburtsstunde der polemischen Schriftgattung De erroribus

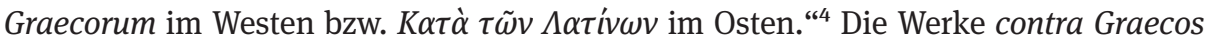
und $\kappa \alpha \tau \dot{\alpha} \tau \tilde{\omega} v \Lambda \alpha \tau i ́ v \omega v$, die diese Linie in unterschiedlichen Stilen, Absichten und Entstehungszusammenhängen jahrhundertelang fortführen, kennen und prägen eine Reihe von Kontroversfragen, die sie je nach Gewichtung und je nach theologischem, ekklesiologischem oder kirchenpolitischem Hintergrund besonders ins Licht rücken. Unter ihnen ragen Themenkomplexe hervor, die zum einen immer wiederkehrende Elemente derartiger Schriften sind, deren Lösung zum anderen in vielen Fällen zur Grundbedingung jedes weiteren Gesprächs oder Austausches gemacht wird: Die richtige, weil rechtgläubige und die kirchliche Tradition seit jeher bewahrende Auslegung jener Themenbereiche ist also konstitutiv für die zu suchende, wiederzufindende oder zu heilende Kircheneinheit. Es sind vor allem vier Themengebiete, die kontroverstheologisches Konfliktpotential bargen und dies zum Teil bis heute im ostwestlichen ökumenischen Dialog tun: Den Bereich der Dogmatik, näherhin den Bereich der Trinitätstheologie wie auch der Pneumatologie berührt die Frage nach dem Ausgang des Heiligen Geistes. Insbesondere die von der Westkirche einseitig vollzogene Einfügung des Filioque in den Wortlaut des nizäno-konstantinopolitanischen Glaubensbekenntnisses und ihre Implikationen gehören $\mathrm{zu}$ den brennendsten und konfliktreichsten Streitpunkten der theologischen Kontroversliteratur spätestens seit dem 11. Jahrhundert. Nicht weniger belastet ist die Frage nach der eucharistischen Zelebration mit gesäuertem oder ungesäuertem Brot, die sogenannte Azymenfrage, womit der Bereich des Ritus und damit der Liturgie betroffen ist. Ein interessanter und in der Analyse ausgewählter Werke zu zeigender Befund ist, dass aber nicht nur die Stellung der Azymenfrage, sondern durchaus auch die im liturgischen Credo gesungene Einfügung des Filioque im Grunde Konfliktstoff ist, der dem Bereich der Liturgie zuzurechnen und von daher sensiblen, weil religiöse Identität stiftenden Parametern unterworfen ist. Auch der dritte Themenkomplex, nämlich der ab dem 13. Jahrhundert aufkommende eschatologische Streit um die Vorstellung des Aufenthaltsortes der Seelen zwischen dem individuellen Tod und dem Jüngsten Gericht (in verkürzter Form oft als Konflikt um das Purgatorium dargestellt) macht deutlich, dass es in den ostwestlichen Auseinandersetzungen in erster Linie Konflikte in Ritusfragen waren, die virulent ausgetragen wurden: ${ }^{5}$ Gerade das in der Liturgie verankerte Gebet für die Verstorbenen wird neben den biblisch-theologischen und patristischen Belegen aus westlicher Perspektive zum Hauptargument für die Existenz eines Reinigungsortes der Seelen der Verstorbenen. Als vierter und besonders in den lateinischen Werken umfassend präsenter Streitpunkt erweist sich die Ekklesiologie, konkretisiert an der

4 CAPIZZI 1999, 267-273, hier: 270 (Hervorhebungen im Original).

5 Y. Avvakumov macht in seiner Studie über Entstehung und Verlauf des Azymenstreits deutlich, dass sich „die gesamten kirchenpolitischen und theologischen Beziehungen zwischen Ost und West im Hochmittelalter in gewisser Hinsicht stets um die Streitfragen des Ritus drehen." (AvvAKUMOV 2002, 16). 
heiklen Frage nach der Vormachtstellung der römischen Kirche innerhalb der oder gar als Universalkirche - ein Konflikt, der die Frage nach der Stellung, den Rechten und dem Vorrang des römischen Papstes inkludiert. Das Thema des päpstlichen Primats ist in vielen Fällen zudem nicht reduziert auf einen Konfliktpunkt unter mehreren, sondern stellt gewissermaßen die Folie aller anderen Divergenzen - seien sie liturgisch, dogmatisch, praktisch usw. - dar: Aus lateinischer Sicht ist die Anerkennung der Vormachtstellung Roms vonseiten der ganzen Kirche, das heißt Lateiner wie Byzantiner, die Bedingung dafür, dass die Einheit der Kirche entweder wiederhergestellt werden kann, sofern sie in den betreffenden Schriften als verloren erachtet wird, oder dass sie sichtbar gemacht werden kann, sofern sie als bestehend, aber gefährdet angesehen wird. So sehr aus lateinischer Perspektive in beiden genannten Fällen das Papsttum an sich der Garant der Einheit der Kirche ist, so wenig herausgehoben ist es aus Sicht der Byzantiner, die dem apostolischen Gleichheitsgedanken bzw. dem Prinzip der Pentarchie folgen, in der es der Theorie und Theologie nach keine Vorrangstellung mit Ausnahme des Ehrenprimats gibt.

Das 13. Jahrhundert stellt nicht nur in kirchenpolitischer Hinsicht eine Ausnahmesituation für die lateinisch-griechischen Beziehungen dar. In vielen Studien, die sich mit den Verhältnissen zwischen den Kirchen im Hoch- und Spätmittelalter befassen, gilt das 13. Jahrhundert im Vergleich zum geradezu irenischen 12. Jahrhundert als die Zeit des endgültigen Bruches, des beginnend-bleibenden Risses zwischen Ostund Westkirche, zum Teil sogar mit scholastisch-offensiver Prägung. ${ }^{6}$ Die vorliegende Studie hat sich vor diesem Hintergrund zum Ziel gesetzt, die diesbezügliche Brisanz des 13. Jahrhunderts und darin vor allem die Zeit des Lateinischen Kaiserreichs von Konstantinopel (1204-1261) unter einem bestimmten, der heutigen ökumenischen Theologie Rechnung tragenden Aspekt zum Thema zu machen: Gegenstand der Untersuchung sind die Beziehungen zwischen der lateinischen und der griechischen Kirche zur Zeit und im geographischen Rahmen des Lateinischen Kaiserreichs (12041261) bis zum II. Konzil von Lyon 1274 (und in begründeten Einzelfällen darüber hinaus). Dabei stehen nicht die kirchenpolitisch-diplomatischen Kontakte im Mittelpunkt, sondern die facettenreiche Darstellung des ost-westlichen theologischen Verhältnisses, wie es im polemischen Schrifttum des 13. Jahrhunderts zum Ausdruck kommt. Im Zentrum der Untersuchung stehen daher ausgewählte literarisch-theologische Werke, die im genannten Zeitraum innerhalb der Reichsgrenzen des Lateinischen Kaiserreichs bzw. des griechischen Exilreichs Nikaia sowohl von lateinischsprachigen als auch von griechischsprachigen Autoren verfasst wurden, aber auch Schriften, die direkten Bezug auf erstgenannte Werke aufweisen. Zeitlich am Beginn des 13. Jahrhunderts anzusetzen und den eingrenzenden Rahmen bis zum II. Lugdunense als dem ersten konziliaren Unionsversuch des Mittelalters zu spannen, bietet sich für das Konzept der vorliegenden Studie aus mehreren Gründen an: Bedingt

6 Zur Haltung der Lateiner gegenüber den Griechen im langen 12. Jahrhundert mit Schwerpunkt auf die kirchenpolitisch-diplomatischen Kontakte vgl. jüngst NEOCLEOUs 2019. 
durch die Ereignisse rund um die Einnahme Konstantinopels 1204 und ihre Implikationen, die wohl am deutlichsten und folgeträchtigsten in der Etablierung des Lateinischen Kaiserreichs zum Ausdruck kamen, ist der „Sitz im Leben“ der betreffenden Schriften ein wichtiger Faktor dafür, was ihnen an theologischem Gehalt entnommen werden kann. Vor dem Hintergrund dessen, dass die Autoren ihre Werke in einer kirchenpolitisch hochbrisanten Zeit verfassten, stellt sich die Frage, ob und inwieweit sich eine derartige Ausnahmesituation auch auf den theologischen Dialog zwischen Ost- und Westkirche übertrug und damit Einfluss auf die theologischen Argumentations- und Begründungsschemata ausübte. Mit anderen Worten: Das Forschungsinteresse der vorliegenden Studie wird von der Frage geleitet, ob und inwiefern sich die kirchenpolitische Ausnahmesituation auch auf die theologischen - heute würde man sagen: ökumenischen - Beziehungen zwischen der lateinischen und der griechischen Kirche, auf ihre Einheitsvorstellungen und -bedingungen sowie auf das Bild auswirkte, das man von der jeweils anderen Kirche hatte.

Im Zentrum der Auswahl literarisch-theologischer Werke, die in der vorliegenden Studie behandelt werden, steht ein herausragendes Werk lateinischer Kontroverstheologie des 13. Jahrhunderts, das nicht nur bestimmend und prägend für eine ganze Reihe nachfolgender Schriften unterschiedlicher Genera wurde, sondern das nach heutigem Wissensstand als erste lateinische Quelle jene Viererstruktur theologischer Kontroverspunkte aufweist, wie sie später prominent auf der Agenda der Konzilien von Lyon 1274 und Ferrara-Florenz 1438/39 zu finden sind: Filioque - Eschatologie/Purgatorium - Azymen - Primat Roms. Es handelt sich um den anonymen Tractatus contra Graecos, der im Jahr 1252 im Dominikanerkonvent von Konstantinopel verfasst wurde und der bisherigen Forschung in Form eines unvollständigen Druckes von zum Teil geringer sprachlicher Qualität in Mignes Patrologia Graeca zur Verfügung stand (PG 140, col. 487-574). In mehr als einer Hinsicht stellt dieses umfangreiche dominikanische Dossier - gleichzeitig Handbuch für lateinische Akteure im Diskurs mit den Byzantinern und Aushängeschild der sprachlichen wie (kontrovers-)theologischen Kenntnisse seines Verfassers - einen Kristallisationspunkt lateinischer Kontroverstheologie dar, die zu entfalten Gegenstand des zentralen dritten Kapitels der vorliegenden Studie ist. Basierend auf der wertvollen und detailreichen Vorstudie von Antoine Dondaine aus dem Jahr $1951^{7}$ liegt jetzt die kritische Edition des Tractatus contra Graecos in der Reihe CCCM (Band 303$)^{8}$ vor, die es ermöglicht, den Traktat solide und in seinen theologischen und historischen Kontext eingebunden darzustellen, wofür wiederum griechisch- und lateinischsprachige Werke des zeitgenössischen theologischen Diskurses exemplarisch ausgewählt und im vierten Kapitel dargestellt wurden.

7 Vgl. Dondaine 1951.

8 Vgl. Anonymus: Tractatus contra Graecos, ed. Andrea Riedl, Turnhout 2020 (= CCCM 303). Sofern nicht anders angegeben, beziehen sich alle Quellenangaben des Traktats auf diese Edition und werden im Folgenden mit TcG abgekürzt. 
Bevor der Aufbau der Studie vorgestellt wird, seien im Hinblick auf die zentrale Analyse der ausgewählten lateinischen und griechischen Werke drei Leitmotive vorangestellt, die das Forschungsinteresse an den Texten leiten:

\section{Erste Vorbemerkung: Polemik}

Die Eingliederung der im Fokus stehenden Werke unter das Stichwort „Polemik“ bzw. „Kontroverstheologie“, wie sie in der Sekundärliteratur durchgehend stattfindet, verlangt zunächst nach einer genaueren Präzisierung dessen, was im jeweiligen Kontext unter Polemik zu verstehen ist. Der Textanalyse vorzuschalten ist daher eine Kriteriologie bzw. Charakterisierung von ost-westlicher Polemik anhand der Auswahl repräsentativer Werke für den gewählten Zeitrahmen. Dies dient in weiterer Folge dazu, einen Leitfaden für die Zuordnung der ausgewählten Werke zum Genre der Polemik zur Hand zu haben und damit ein Prüfinstrument, ob und inwieweit als Polemik geltende Schriften den Kriterien dieses Genres entsprechen. Daran anschließend sollen im Zuge der einzelnen Werkanalyse die Auswirkungen berücksichtigt werden können, die das „Gewand der Polemik“ auf die transportierten theologischen Inhalte hat.

H. Smolinsky stellt in seinem Artikel über „Kontroverstheologie“ im Lexikon für Theologie und Kirche kritisch fest, dass zu klären sei, ob und inwieweit die „Auseinandersetzungen mit der Ostkirche, die oft auf eine Kirchenunion zielten“"9, als Kontroverstheologie zu qualifizieren seien. Seine kritische Anfrage impliziert, dass das Streben nach der Kircheneinheit ein Ausschließungsgrund polemischer Intentionen sei. Oder, umgekehrt und allgemeiner formuliert: Polemische Methoden verfolgen vielmehr eo ipso nicht das Ziel einer Einigung in der diskutierten Angelegenheit. Gerade vor dem Hintergrund dieses am Einzelfall zu klärenden Verständnisses von Polemik in der ost-westlichen Kontroverse lohnt der Blick auf eine Auswahl repräsentativer Sekundärliteratur, die sich mit ost-westlicher Kontroverstheologie des Mittelalters befasst und entweder in Randbemerkungen erwähnt oder - seltener - eine klare Definition dessen voranstellt, was sie im jeweiligen Kontext unter Polemik versteht:

H. G. Beck vertrat in der Mitte des 20. Jahrhunderts in seinem vielzitierten Standardwerk Kirche und theologische Literatur im byzantinischen Reich (Erstauflage 1959) die Sichtweise, dass die Dogmengeschichte der byzantinischen Kirche ,in erster Linie die Geschichte der byzantinischen Polemik“10 sei, dass die byzantinische Dogmatik „sich in einem hohen Maße in der Polemik erschöpft.“11 Aus diesem Blickwinkel betrachtet entsteht die Dogmatik als Lehrgebäude der Kirche aus dem polemischen

9 SMOLINSKY 1997, 333-335, hier: 335.

10 BECK 1959, 279. Als explizite Reaktion auf diese Sichtweise vgl. den Sammelband Rigo/ERmiLOV 2009.

11 BECK 1959, 279. 
Diskurs bzw. kann - radikal formuliert - als dessen Produkt gesehen werden. Beck gesteht allerdings auch „Lücken [ein], welche die Polemik offenlässt“"12, die zu finden und zu überbrücken sind. Ohne an dieser Stelle auf die Implikationen jener Sichtweise für die byzantinische Dogmatik einzugehen, ergibt sich als Indiz seines Verständnisses von theologischer Polemik, dass polemisches Agieren ein Profilierungsinstrumentarium darstellt, mittels dessen man in Abgrenzung von und Schärfung an einem Gegenüber das artikulieren kann, was das Eigene ist, was als das zu Vertretende angesehen wird und entsprechend argumentiert bzw. verteidigt werden muss. Nach der Definition von Ch. Schabel dagegen ist ein Text dann als polemisch zu charakterisieren, wenn der Autor Thema und Agenda seines Werkes mit dem Ziel wählt, entweder dem Gegner inhaltlich und verbal überlegen zu sein oder dem eigenen Kreis Argumente für die Debatte mit dem Gegner zu liefern. ${ }^{13}$ Y. Avvakumov grenzt in seiner Studie Die Entstehung des Unionsgedankens polemische Literatur von Werken ab, die sich mit der kirchenrechtlichen Dimension (Dekretalien und Konzilsdokumente) und der Lehre bzw. Theologie der Kirche (systematisch-theologische Abhandlungen) befassen, da diese nicht in Abgrenzung, sondern in positiver Darstellung ,den allgemeingültigen Fundus des theologischen Wissens und Könnens" ${ }^{114}$ vermitteln. Er unterteilt zudem die Schriften, die sich mit den Kontroversthemen zwischen Byzantinern und Lateinern beschäftigen, in zwei Kategorien: zum einen Schriften, „deren Haupt-

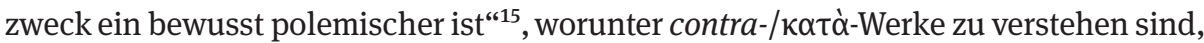
außerdem Berichte über mündliche Disputationen, Briefe als Handreichung und Zitatensammlung sowie Abhandlungen, die als direkte Reaktion auf Polemik entstanden sind. Zur zweiten Kategorie zählt Avvakumov Werke, deren Absicht „primär unpolemisch“16 ist und die die Frage nach der Beziehung untereinander „vor allem illustrativ, aber auch argumentativ" ${ }^{\prime 17}$ berühren. C. Delacroix-Besnier, die sich in ihren Studien hauptsächlich den im Osten angesiedelten Mendikantenorden in der Auseinandersetzung mit der griechischen Kirche widmet, stellt die beiden Begriffe Polemik und Dialog einander gegenüber und definiert damit Polemik nicht als Methode, sondern als Medium der Kommunikation: Während der Dialog mündlich und in direktem Zusammentreffen stattfindet, handle es sich bei Polemik um die einseitige Positionierung in der Auseinandersetzung mit dem Gegenüber in verschriftlichter Form. ${ }^{18}$ Insofern spricht sie an vielen Stellen von der Ausbildung und Etablierung

12 Ebd.

13 Vgl. SCHABEL 2011, 85-128, hier: 89.

14 Avvakumov 2002, 18.

15 Ebd., 117. Avvakumov zählt auch Humbert von Romans zu den Polemikern.

16 Ebd.

17 Ebd.

18 Delacroix-Besnier 2011, 151-168, hier: 151. 
einer „tradition polémique“19 der Dominikaner bzw. generell der Mendikanten im Osten. M.-H. Blanchet bietet eine umfangreiche und methodenfokussierte Definition von Polemik in Bezug auf kontroverstheologische Literatur, die sie am Beispiel des Dialogue avec un Moine contre les Latins (1442) des Théodore Agallianos illustriert: Instrumente bzw. Stilmittel der Polemik seien die bewusste Vermischung von Themen und Zugangsweisen, die missbräuchliche Verallgemeinerung, Böswilligkeit und Unaufrichtigkeit in der Argumentation, Wortspiele und bewusste Sinnentstellungen, Schmähung und Beschimpfung sowie die Belustigung auf Kosten des Gegenübers. ${ }^{20}$ A. Riebe versteht in ihrer Studie über Johannes XI. Bekkos Rom in Gemeinschaft mit Konstantinopel eine „scharf und profiliert kontroverstheologisch[e]“ Ausrichtung eines Textes als Gegenteil eines Tons, der „vermittelnd-versöhnlich ist“21. A. Dondaine schließlich benennt in seiner zum Standardwerk gewordenen Studie Contra Graecos ein polemisches Werk klassisch als ein „oeuvre apologétique“22.

Vor dem Hintergrund dieser Sammlung von Merkmalen der Polemik innerhalb der Ost-West-Kontroverse sind es vor allem drei Elemente, die als Gemeinsamkeit herausgefiltert werden können: Erstens setzen sich polemische Werke auf unterschiedliche Weise mit einem - realen oder fiktiven - Gegenüber auseinander und sind in Abgrenzung zu diesem verfasst. Zweitens zielen polemische Werke nicht auf einen Konsens oder auf Kompromisslösungen, sondern konzentrieren sich auf die Verteidigung und/oder Vermittlung des Eigenen, wobei die Methoden entsprechend angepasst werden. Dies führt dazu, dass es nicht das Ziel eines Disputs mit dem Gegenüber ist, seine inhaltlichen Entfaltungsmöglichkeiten im Sinne eines konstruktiven Dialogs $\mathrm{zu}$ fördern, sondern dass das Ziel einer solchen Auseinandersetzung von vornherein festgelegt ist. Anders als bei einer Darstellung des „allgemeingültigen Fundus des theologischen Wissens und Könnens“23 in positiver Weise, wie Y. Avvakumov formuliert, geht es darum, siegreich aus einer Debatte hervorzugehen und die Mittel an die bestmögliche Erreichung dieses Erfolges anzupassen. Ein drittes Merkmal zur Charakterisierung von Polemik ist das bewusste Einsetzen des Argumentum ad hominem, das heißt die Diffamierung bzw. Abwertung der Meinung, der Expertise oder allgemein der Person des Gegenübers, die damit als gegenstandslos, belächelnswert oder gar frevlerisch dargestellt wird und deren Meinung auf diesem Hintergrund umso mehr einer Korrektur und Rückführung bedarf. Entsprechende Stilmittel in der Argumentation und abwertende Zuschreibungen bis hin zu Beleidigungen kreieren damit ein Ungleichgewicht der am Disput - auch wenn nur fiktiv - Beteiligten, das

19 Vgl. den Titel des fünften Kapitels (et passim) der unveröffentlichten Habilitationsschrift von DELACROIX-BESNIER 2007. Ich danke Chris Schabel, dass er mir das Manuskript zur Verfügung gestellt hat.

20 BLANChet 2013, 114-115.

21 Riebe 2005, 293.

22 Dondaine 1951, 428.

23 Avvakumov 2002, 18. 
analog zum zweiten Charakteristikum auf eine Stärkung und Unterfütterung der eigenen Position zielt.

Merkmale von Polemik sind demnach: a) Auseinandersetzung in Form von Abgrenzung; b) kein/e Konsens/Konvergenz/Kompromiss, sondern Orientierung daraufhin, sieg- bzw. erfolgreich aus einer Debatte oder einer Konfrontation hervorzugehen; c) Anpassung der Mittel zur Erreichung dieses Zieles. Die Analyse der im Fokus der vorliegenden Studie stehenden Schriften wird jedoch zeigen, dass die Rede von einer „polemischen Methode“ im Sinne einer Tonart jeglicher Kommunikation der differenzierten Herangehensweise von Autoren, deren Werke als als Polemik gelten, nur zum Teil gerecht wird. Oder anders formuliert: Dass sich die Auseinandersetzung der Autoren mit ihrem Gegenüber unter dem Mantel der Polemik als erstaunlich vielfältig und einfallsreich erweist, wird bei einer ersten Qualifizierung als Polemik oft übersehen. Es wird daher für das Konzept der vorliegenden Studie notwendig sein, das jeweils hinter den (polemischen) Methoden stehende Kirchenbild und die mit ihm korrespondierenden kirchlichen Einheitsvorstellungen der Autoren herauszufiltern und so ein dezidiert theologisches Motiv freizulegen, das zusätzlich zum theologiegeschichtlichen Interesse das Anschlusspotential an die heutige ökumenische Theologie bzw. an den gegenwärtigen ökumenisch-theologischen Dialog gewährleisten kann.

\section{Zweite Vorbemerkung: Methodik}

Besonders mit den Namen J. Meyendorff und A. Papadakis ist die These verbunden, dass die Methodik der mittelalterlichen Autoren im sichtbaren Niederschlag ihrer Werke im kontroverstheologischen lateinisch-griechischen Kontext wesentlich dafür verantwortlich war, dass die Kommunikation nicht gelingen konnte oder sogar scheitern musste - eine These, der M. Plested prominent in seiner Studie Orthodox Readings of Aquinas widersprochen hat. Das Scheitern, so die These, sei darin ersichtlich, dass das Ziel einer Kirchenunion nicht erreicht wurde bzw. dass man einander erst gar nicht verstand, weil die Herangehensweise an theologische Debatten und damit der theologische Bildungshintergrund - grundlegend verschieden war. ${ }^{24}$ Ausgehend von der Feststellung, dass der diesbezügliche Kontrast zwischen Ost und

24 Vgl. Papadakis 1994, 151-198, hier: 167-187 und bes. 181-187; vgl. MeyendoRfF 1984; vgl. MEYENDORFF 1988, 395-407, hier: 396-397; vgl. Plested 2012. Es ist auffällig, dass Meyendorff gerade zu den Verhandlungen von Nikaia/Nymphaion bzw. zum neuen Verhandlungsthema des Purgatoriums in Lyon 1274 die neuen Erkenntnisse A. Dondaines nicht zitiert. Die Studie von Dondaine muss er dennoch gekannt haben, da er sie als Beleg dafür heranzieht, dass Thomas von Aquin den Auftrag erhalten habe „ein anti-griechisches Dossier für das Konzil von Lyon vorzubereiten“ (406), womit er den Ausführungen Dondaines allerdings nicht gerecht wird. 
West geradezu eklatant war ${ }^{25}$, sieht A. Papadakis den ausschlaggebenden methodischen Unterschied darin, dass der Westen sich mit dem Aufkommen und Erstarken der Scholastik und generell des schulgebundenen - und damit systematischen - wissenschaftlichen Arbeitens und Denkens sukzessive von der Väter-Theologie (ad mentem patrum) distanzierte, während der Osten weiterhin daran festhielt, im Geiste und in den Spuren der Väter Theologie zu betreiben. Die byzantinische Theologie habe sich im Kontrast zur westlichen aus Patristik und Gebet gespeist und wurde nicht durch Schule und Lehre, sondern in erster Linie durch Predigt vermittelt. ${ }^{26}$ Für A. Papadakis ist (unter Bezugnahme auf die Forschungen J. Meyendorffs) der bildungstheoretische und damit die Methodik der Werke beeinflussende Unterschied derart dominant, dass er ihn zusammen mit den päpstlichen Primatsansprüchen zum hauptsächlichen Grund des Bruchs zwischen Ost- und Westkirche stilisiert. ${ }^{27}$ Seinen Niederschlag findet dieser postulierte Unterschied, so die These, in Stil und Methode der kontroverstheologischen Werke: Während die im Westen ausgebildeten und in der lateinischen Theologie versierten Autoren argumentative Traktate zur Verteidigung oder Erläuterung der lateinischen Positionen im Sinne einer Handreichung, eines Gutachtens oder schlicht einer theologischen Abhandlung verfassten, seien die Werke der griechischen Autoren zu einem großen Teil Katalog- oder Listenschriften, die gegebenenfalls, aber keineswegs immer unter Rückgriff auf patristische Autoritäten schlicht die Irrtümer der Lateiner aufzählen, ohne dabei Interesse an deren Wider-

25 S. Ebbesen nennt die westlichen Theologen „scientists“, die geprägt von der scholastischen Methode aus dem Fundus eines theologischen Studiums schöpften, das die Aktualität und den Stand der Wissenschaft der neu gegründeten Bildungseinrichtungen im Westen spiegelte. Die Griechen bezeichnet er als „humanists“, die mehr auf die Bewahrung und Fortführung althergebrachter, klassischer Methoden und weniger darauf bedacht waren, kohärente Argumentationsgänge zu entwickeln; vgl. EBBESEN 2011, 169-182, hier: 170. J. Meyendorff hingegen stellt wenig differenziert die „professional ,scholastics“ of the West“ den "old-fashioned sophisticated scholars of Byzantium“ gegenüber (MEYENDORFF 1988, 406); vgl. auch PodSKalsKy 2003; vgl. HusSEy 1986, 4.

26 Vgl. PAPADAKIS 1994, 181-183; „Byzantine theology was a continuation of the patristic legacy, and $\mathrm{a}[\mathrm{s}] \mathrm{such}$, was learned primarily by the reading and hearing of Scripture and of course by praying. It was never at any rate pursued as a purely rationalistic activity. [...] This theology could only be 'preached' or 'proclaimed', and not be simply 'taught' in a school manner." (Ebd., 182-183; Papadakis paraphrasiert MEYENDORFF 1984, 68).

27 ,The change in methodology introduced in the West by scholasticism was to make theological exchange with the East rather difficult. Time and again the western complaint was to be that the Orthodox East was incapable of theologizing professionally or argumentatively. On the other hand, Byzantine churchmen could not understand how theology could be viewed as a rational discipline; [...] To be short, the fundamental reorientation of western theology in the twelfth century, along with the papal Petrine claims, must be viewed as factors contributing to the disruption of Christendom. Both scholasticism and the Roman primacy, in a sense, changed the rules of the game and, as a consequence, destroyed 'living continuity with the common past of the Church universal'. The synchronous development of Latin scholasticism and schism at any rate was not a purely historical accident.“ (PAPADAKIs 1994, 184). 
legung oder Korrektur zu haben. ${ }^{28}$ Dass die methodischen - und dadurch begründet

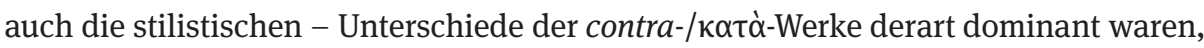
dass sie das gegenseitige Verstehen beeinträchtigten oder gar verunmöglichten, biete außerdem eine Grundlage für die Annahme, dass es den jeweiligen Autoren ohnehin nicht darum ging bzw. dass gelingende Kommunikation - anders als etwa in den diplomatischen Verhandlungen - ohnehin nicht intendiert gewesen sei. ${ }^{29}$

Die vorliegende Studie folgt in der Analyse und unterschiedlichen Gewichtung ausgewählter Quellen des 13. Jahrhunderts dem von M. Plested eingeschlagenen Weg: Zunächst soll gerade die Frage nach dem methodischen Unterschied zwischen Werken lateinischer und byzantinischer Herkunft und - sofern ein solcher Unterschied tatsächlich oder tendenziell besteht - nach seinen Implikationen auf das gegenseitige Verstehen mittels einer Analyse des Stils und der Methodik des betreffenden Schriftencorpus erhellt werden, um zu quellenfundierten Aussagen zu kommen. Es sei vorweggenommen, dass die Grenzen dieses postulierten Unterschiedes oft ineinander verschwimmen: Gerade hinsichtlich der Argumentationsweisen finden sich prominente Beispiele dafür (so etwa der im Fokus der vorliegenden Studie stehende dominikanische Tractatus contra Graecos aus dem Jahr 1252), dass geradezu bewusst die Methode des Gegners angewandt wurde, um in den Verstehenshorizont des Gegenübers eindringen und ihm so die Plausibilität der eigenen Argumente aufzeigen zu können. Das Gelingen oder Scheitern der theologischen Debatten kann daher weder ausschließlich noch vorrangig auf stilistische und methodische Unterschiede gemünzt werden. Dass der päpstliche Primatsanspruch zum Stein des Anstoßes in den theologischen lateinisch-griechischen Beziehungen wurde bzw. welchen Rang jener Konfliktpunkt in der theologischen Agenda des 13. Jahrhunderts einnahm, soll für die betreffenden Texte eingehend anhand des Motives des Kirchenbildes untersucht werden. Dazu bedarf es einiger Vorverständnisse und Klärungen von Begrifflichkeiten - kurz: es bedarf der Koordinaten des Kirchenbildes -, die als Vorzeichen für die folgenden, auf das gewählte Schriftencorpus bezogenen Ausführungen über deren jeweiliges Kirchenbild Geltung haben und allgemein für die Leitfrage der vorliegenden Studie grundlegend sind.

28 Für einen Überblick über die (quantitativ gelisteten) Inhalte griechischer Irrtumslisten vgl. KoLBABA 2000.

29 Vgl. etwa die Ansicht S. Burkhardts, dass es „das Hauptproblem der Kirchenunion war, dass sie von einem Großteil der orthodoxen Kirche und der orthodoxen Christen nicht gewünscht wurde.“ (BURKHARDT 2014, 266). 


\section{Dritte Vorbemerkung: Das Motiv des Kirchenbildes}

Das Motiv des Kirchenbildes ist generell ein weit gefasstes und berührt zunächst hauptsächlich ekklesiologische Fragestellungen ${ }^{30}$, ohne darauf beschränkt zu bleiben. Es sei mit einem systematischen Zugang begonnen: Mit dem Thema des Kirchenbildes in Geschichte und Gegenwart bzw. in unterschiedlichen Zugängen beschäftigte sich der Dogmatiker W. Beinert gemeinsam mit Kollegen der Fakultät für Katholische Theologie in Regensburg: Als Herausgeber verpackt er sowohl die Ausgangsfrage nach der Kirche als auch den Grundtenor einer vielgestaltigen Antwort in den prägnanten Titel des Sammelbandes: Kirchenbilder - Kirchenvisionen. Variationen über eine Wirklichkeit und stellt das Thema der darauffolgenden Beiträge folgendermaßen vor: „Die Frage nach der Kirche: Variationen über eine Wirklichkeit erhalten wir anstelle der einen Antwort. “31 Wenn von Kirchenbildern die Rede ist, sei zunächst zu klären, welche Wirklichkeit das jeweilige Bild abbilden will und soll: Soll die Frage nach dem Wesen der Kirche, nach dem „Verhältnis von Mysterium und Institution“ beantwortet werden (Was ist die Kirche?), geht es um die Frage nach „ihren Strukturen, ihrer Verfassung und dem Zusammenleben ihrer Mitglieder“ (Wie lebt die Kirche in der Zeit?) oder steht die „Plausibilität von Leben und Verkündigung der Kirche“32 im Vordergrund (Wie präsentiert sich Kirche?)? Da es das verbindende Ziel aller in der vorliegenden Studie zu analysierenden Werke ist, in Abgrenzung zu einem Gegenüber das zum Ausdruck zu bringen, was als das Proprium und Charakteristikum der wahren Kirche angesehen wird, können und müssen sowohl die Grenzen als auch die Spielräume des Kirchenbildes von dorther definiert werden: Wo die Texte Einblick in das Wesensbild der Kirche geben, soll dies zur Darstellung gebracht werden. Wo das Wesen der Kirche nicht im Brennpunkt steht, sondern mehr das theologische Gerüst für die Argumentation der Kirchenstrukturen bildet, soll der Fokus auf letzteren liegen. E. von Ivánka formuliert mutig, dass es bezüglich des Kirchenbegriffs, das heißt hier konkret bzgl. der Frage nach dem Wesen der Kirche, zwischen östlicher und westlicher Tradition keine Uneinigkeiten oder Unterschiede gegeben habe. Die Vorwürfe auf beiden Seiten würden „keineswegs vom Standpunkt einer anderen Ekklesiologie“ herrühren, sondern hätten „mit kirchenrechtlich-hierarchischen Argumenten $^{\text {“33 }} \mathrm{zu}$ tun. Für die folgende Darstellung des Kirchenbildes der ausgewählten Werke

30 Vgl. die traditionelle, zweifache Zuordnung der Ekklesiologie innerhalb des theologischen Fächerkanons: Zum einen der Fundamentaltheologie insofern, als ihr „die kritische Frage nach der Wahrheit der Kirche im Dienst der Vermittlung des Offenbarungsgeschehens [obliegt]: Welche Bedingungen müssen erfüllt sein, dass die Kirche mit Recht den Anspruch auf wahre und verbindliche Weitergabe des geoffenbarten und geglaubten Wortes Gottes erheben kann?"; zum anderen der Dogmatik insofern, als es ihr um das „Geschehen der vom Heiligen Geist ermöglichten Annahme und Vergegenwärtigung des in Jesus Christus mitgeteilten Lebens Gottes durch die Gemeinschaft der Glaubenden“ geht; beide Zitate aus KeHL 1995, 568-573, hier: 569.

31 BEINERT 1995, 10 (Vorwort).

32 Alle Zitate aus BEINERT 1995a, 58 -127, hier: 62.

33 Beide Zitate aus von IvÁNKA 1961, 407-429, hier: 412. 
wird zu prüfen sein, inwieweit es tatsächlich auf den zweiten der drei Aspekte, die W. Beinert anführt, zu beschränken bleibt: Auf das Kirchenbild der „Kirche in der Zeit“, auf Struktur, Verfassung und Zusammenleben und somit auf das, was W. Beinert das „ekklesiologische Zentralthema des Mittelalters“34, was E. von Ivánka die „hierarchisch-autoritative Kirchenauffassung“35 nennt.

In den im Fokus stehenden Werken kann durchgehend beobachtet werden, dass die Einheit der Kirche bildlich gesprochen der Schauplatz der Auseinandersetzungen ist. Sie bildet je nach Gewichtung des einzelnen Textes gleichzeitig den Rahmen und den Gegenstand des ost-westlichen Konflikts: den Rahmen insofern, als unter dem Postulat der Einheit der Kirche die je eigene Tradition als die richtige, die traditionsbewahrende ins Feld geführt wird, die somit als die Repräsentantin der kirchlichen Einheit von allen anzuerkennen ist (= die Kirche ist ja eine Kirche). In diesem Zusammenhang spielt das Stichwort ,Wahrheit‘ bzw. die wahre Kirche - geknüpft an die Einheit, die die Wahrheit garantiert - eine präsente Rolle in den Auseinandersetzungen. Gegenstand des Konflikts ist die kirchliche Einheit insofern, als es letztlich das Ziel der Akteure ist, für eine solche kirchliche Einheit zu argumentieren, die im 13. Jahrhundert mit den Implikationen des Pontifikats Innozenz III. erstmals als verloren angesehen wurde ${ }^{36}$ (= die Kirche soll ja eine Kirche sein). Eine derartige Argumentation für die kirchliche Einheit findet sich allerdings - anders als z.B. in den Werken des Patriarchen von Konstantinopel Johannes XI. Bekkos ${ }^{37}$ - seltener in offenkundiger und ausgewiesener Art, sondern darin, das der Einheit entsprechende ,Richtige‘ im Vergleich zum ,Falschen“ des Anderen herauszustellen und die Einheit auf diese Weise plausibel, ja zwingend zu machen. Anders formuliert läuft es letztlich darauf hinaus, dass die Einheit dann verwirklicht und garantiert ist, wenn das sie bedrohende Schisma entlarvt und seine Protagonisten vor die Wahl gestellt sind, zur Einheit zurückzukehren oder - wegen unnachgiebigen Festhaltens am Falschen - der Häresie anheimzufallen, was den Ausschluss aus der kirchlichen Einheit zur Folge hat. Einheit bedeutet also in keinem der Fälle eine Summe, einen Zusammenschluss aus vorher eigenständigen oder eigenständig gewordenen Elementen, sondern das

34 BEINERT 1995a, 62.

35 VON IVÁNKA 1961, 412.

36 Vgl. Avvakumov 2002, 86; vgl. Schabel 2008, 320 - 335, hier: 320. - Eine These, die im Einzelnen zu prüfen ist: Die literarisch-theologischen Schriften, die in der vorliegenden Studie im Fokus stehen, sollen daraufhin untersucht werden, ob und wenn ja, mit welcher Begründung sie die kirchliche Einheit als verloren ansehen. In Bezug auf die lateinischen Schriften ist diese Frage insofern von besonderem Interesse, als der Westen bzw. Innozenz III. ja gerade das Vierte Laterankonzil (1215) als Bestätigung der ost-westlichen Einheit der Kirchen verstanden hat. In Bezug auf die griechischen Schriften findet sich in der Sekundärliteratur immer wieder der Schluss, die Byzantiner hätten kein Interesse bzw. keinen Leidensdruck an den Tag gelegt, der als Motor einer solchen Suche nach der kirchlichen Einheit fungieren würde.

37 Vgl. die ausgezeichnete Studie von A. Riebe über Johannes XI. Bekkos als Verteidiger der Kirchenunion von Lyon 1274 (RIEBE 2005). 
Bleiben in einer oder Zurückkehren in eine Wirklichkeit, die nach den jeweils eigenen Kriterien als eine solche Einheit betrachtet wird. ${ }^{38}$

Die Koordinaten der $\mathrm{zu}$ analysierenden Kirchenbilder sind zusammenfassend folgende: Im Fokus stehen die kirchlichen Strukturen (sowohl Leitungs- als auch generelle Legitimationsstrukturen von kirchlicher Wirklichkeit), die der jeweilige Autor als Kennzeichen der einen und deswegen wahren Kirche ansieht. In der Regel wird die Darstellung dieser Strukturen von dem genährt, was nach dem eigenen kirchlichen Verständnis als Defizit des Gegenübers gewertet wird, was diesem also fehlt oder gar falsch ist. ${ }^{39}$ Auf diese Weise wird nicht nur das Bild der eigenen Kirche deutlich, sondern - ex negativo oder in seltenen Fällen ausdrücklich - auch das Kirchenbild des Gegenübers. Die Rolle, die die ekklesiologische Folie, das heißt die vorherrschende lehramtliche Kirchen- und/oder Primatsauffassung, dabei jeweils spielt, soll ebenso veranschaulicht werden wie die Argumentationsstrategien des Autors zur Verteidigung dieses bzw. des eigenen Kirchenbildes.

Da die zu analysierenden Werke zwar allesamt den Entstehungshintergrund der ost-westlichen theologischen Kontroverse aufweisen, da sie zu einem Großteil dem Genre der Kontroverstheologie bzw. der Polemik zuzuordnen sind, aber dennoch verschiedenen unmittelbaren Kontexten entspringen und vielgestaltige Absichten verfolgen bzw. Verwendungszwecke widerspiegeln, behält sich die Verfasserin der vorliegenden Studie vor, die Struktur der Werkanalyse gelegentlich den individuellen Inhalten anzupassen. ${ }^{40}$ In Grundzügen aber erfolgt die Darstellung in drei Schritten: Nach einem einleitenden Inhaltsüberblick zur Verortung der Kontroversthemen im jeweiligen Werk liegt der Fokus zunächst auf der Basis der Argumentation, das heißt auf den Verfahren, die die Autoren zur Erreichung ihrer Ziele anwenden, die auf diese Weise ebenfalls zur Darstellung gelangen. Im Anschluss daran soll anhand dreier Leitfragen auf das Motiv des Kirchenbildes eingegangen werden, wie es oben vorgestellt wurde:

1) Welches Kirchenbild bringt der Autor mit und macht es zum Gegenstand seiner Verteidigung?

38 Th. Hofmann polarisiert zwei exklusive Modelle der Kircheneinheit: „Während Rom als entscheidenden Akt [einer Kirchenunion] die Rückkehr der Griechen in den Schoß der einen Kirche, die natürlich durch Rom repräsentiert wurde, ansah, betonte Byzanz das Nebeneinander von zwei Kirchen, die trotz Abweichungen beide als rechtgläubig zu bezeichnen sind“ (HofmANN 1995, 103. Hervorhebungen im Original.). A. Riebe hingegen streicht die „bemerkenswerte Richtung“ heraus, nach der für Johannes XI. Bekkos als Repräsentant der griechischen Seite die Wiederherstellung der Einheit funktioniert: „Es ist die östliche Kirche des neuen Rom, die Gemeinschaft aufnimmt mit der Kirche Altroms. Bekkos redet nie anders. Immer ist es die eigene Kirche, die die römische Kirche als nicht häretisch akzeptiert und mit ihr - wieder - Gemeinschaft hält.“ (RIEBE 2005, 146).

39 Vgl. dazu H. Petri, der diese Vorgehensweise für heutiges ökumenisch-kirchliches Handeln als Negativfolie anführt (PETRI 1995, 128-157, hier: 151).

40 In manchen, besonders die griechischen Werke betreffenden Fällen ist die Wahl dieses Vorgehens auch dem mangelhaften Text- bzw. Editionsbestand geschuldet. Wo dies der Fall ist, wird eigens darauf hingewiesen. 
2) Mit welchen Argumentationsstrategien verteidigt er dieses Kirchenbild?

3) Was sagt die Analyse von 1) und 2) über das Bild des Autors von der jeweils anderen Kirche aus?

\section{Zum Aufbau der vorliegenden Studie}

Um die im Fokus stehenden lateinischen und griechischen Werke entsprechend zu kontextualisieren und sie, soweit rekonstruierbar, ihrem „Sitz im Leben“ zuordnen zu können, sollen zu Beginn die kirchenpolitischen Rahmenbedingungen und Entstehungszusammenhänge dargelegt werden: Das erste Kapitel befasst sich vor diesem Hintergrund mit dem Vierten Kreuzzug (1202-1204), der Einnahme Konstantinopels (1204) und der Etablierung lateinischer - weltlicher wie kirchlicher - Herrschaftsstrukturen in Gestalt des Lateinischen Kaiserreichs von Konstantinopel (1204-1261). Die Haltung des kirchlichen Westens, die Geburt der byzantinischen Exilreiche und das Lateinische Patriarchat von Konstantinopel stellen erste Schwerpunkte der Ausführungen in diesem Kapitel dar, die so gewichtet sind, dass sie auf die Einbettung der literarisch-theologischen Werke in ihren Zeitkontext zielen. Im Sinne einer Kanalisierung auf die theologischen Konfliktpunkte hin folgt die Darstellung der Stationen der Unionsbemühungen des 13. Jahrhunderts: Darunter sind die diplomatischen Kontakte subsumiert, das heißt die Gesandtschaften in beide Richtungen mit dem Ziel, Einigkeit und Klärung zu schaffen, außerdem die großen konziliaren Etappen, das heißt die Thematisierung der Griechenfrage auf den Konzilien des 13. Jahrhunderts, und schließlich eine theologisch-inhaltliche Hinführung zu den im Fokus stehenden literarisch-theologischen Werken.

Das zweite Kapitel trägt der Schwerpunktsetzung auf den anonymen Tractatus contra Graecos (1252) Rechnung, indem es der Präsenz des Dominikanerordens im Osten in lokaler, edukativer und literarischer Hinsicht gewidmet ist. Erste Notizen zu diesem kontroverstheologischen Dossier als Produkt dominikanischer Präsenz in der Provinz Graecia bzw. zu dessen Sonderstellung in der lateinisch-griechischen literarischen Kontroverse leiten über zu den beiden folgenden Kapiteln, die die konkrete Werkanalyse zum Gegenstand haben.

Das dritte und vierte Kapitel sind der eigentliche Schwerpunkt der Studie, nämlich die Analyse der betreffenden Werke - zunächst der lateinischen, dann der griechischen - nach den in den Vorbemerkungen thematisierten Kriterien. Ein in vielerlei Hinsicht aufschlussreicher Briefwechsel zwischen dem griechischen Patriarchen im Exil, Johannes X. Kamateros, und Papst Innozenz III. in den Jahren 1198 und 1199 stellt gewissermaßen die Folie dar, auf die die Inhalte der lateinischen und griechischen Schriften gelegt und nach Kontinuität oder Innovation hin geprüft werden sollen. Für die lateinische Seite fällt die Analyse des Tractatus contra Graecos des anonymen Dominikaners im dritten Kapitel am umfassendsten aus, weil dieses Dossier als lateinisches Modell und Veranschaulichung einer Arbeitsweise dient, die - zumindest in der polemischen Tradition der Dominikaner des Ostens - als eine sich bewährende 
vielfach aufgegriffen wurde. Es folgen im vierten Kapitel die Analyse des Libellus des Nikolaos von Cotrone, des Gutachtens Contra errores Graecorum des Thomas von Aquin sowie ein Exkurs auf seine kurze Schrift De rationibus fidei ad cantorem Antiochenum, des Opus tripartitum des Humbert von Romans, der beiden Werke Thesaurus veritatis fidei und Contra Graecos des Bonacursius von Bologna und der Revision des Tractatus contra Graecos durch Bartholomeus Constantinopolitanus. Auf griechischer

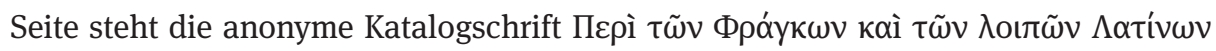
bzw. Opusculum contra Francos chronologisch am Beginn der Werkanalysen. Ihr folgt

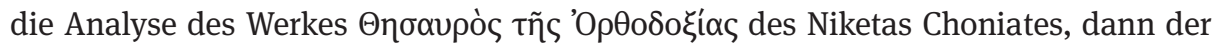

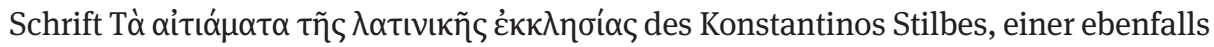

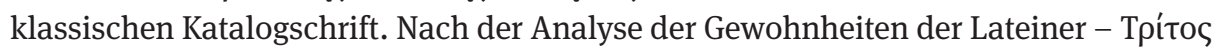

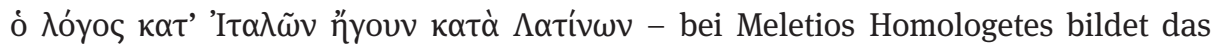

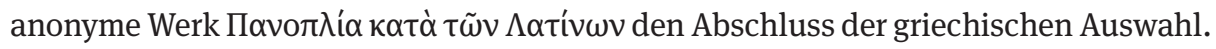

Ein fünftes und abschließendes Kapitel bringt die Ergebnisse der Werkanalysen in eine Synthese, prüft sie anhand der eingangs gestellten Kriterien und formuliert ihre Implikationen auf die literarisch-theologischen Beziehungen zwischen Ost- und Westkirche zur hochbrisanten Zeit des Lateinischen Kaiserreichs in Konstantinopel. 